\title{
Characterization of BoaCRTISO Reveals Its Role in Carotenoid Biosynthesis in Chinese Kale
}

\author{
Min Jiang ${ }^{1 \dagger}$, Fen Zhang ${ }^{1 \dagger}$, Qiao Yuan', Peixing Lin' ${ }^{1}$, Hao Zheng ${ }^{1}$, Sha Liang ${ }^{1}$, Yue Jian', \\ Huiying Miao ${ }^{2}$, Huanxiu $\mathrm{Li}^{1}$, Qiaomei Wang ${ }^{2 *}$ and Bo Sun ${ }^{1 *}$ \\ ${ }^{1}$ College of Horticulture, Sichuan Agricultural University, Chengdu, China, ${ }^{2}$ Key Laboratory of Horticultural Plant Growth, \\ Development and Quality Improvement, Ministry of Agriculture, Department of Horticulture, Zhejiang University, Hangzhou, \\ China
}

\section{OPEN ACCESS}

Edited by:

Renata Rivera-Madrid, Scientific Research Center of Yucatán

(ClCY), Mexico

Reviewed by:

Tianhu Sun,

Cornell University, United States

Sarah Frusciante,

Italian National Agency for New Technologies, Energy and Sustainable Economic Development (ENEA), Italy

*Correspondence:

Qiaomei Wang

qmwang@zju.edu.cn

Bo Sun

bsun@sicau.edu.cn

${ }^{\dagger}$ These authors have contributed equally to this work

Specialty section:

This article was submitted to Plant Metabolism

and Chemodiversity,

a section of the journal

Frontiers in Plant Science

Received: 15 February 2021 Accepted: 09 April 2021

Published: 14 May 2021

Citation: Jiang $M$, Zhang $F$, Yuan $Q$, Lin $P$, Zheng $H$, Liang S, Jian Y, Miao $H$,

Li H, Wang Q and Sun B (2021)

Characterization of BoaCRTISO

Reveals Its Role in Carotenoid

Biosynthesis in Chinese Kale.

Front. Plant Sci. 12:662684.

doi: 10.3389/fp/s.2021.662684
Carotenoids are organic pigments that play an important role in both plant coloration and human health; they are a critical subject in molecular breeding due to growing demand for natural molecules in both food and medicine. In this study, we focus upon characterizing BoaCRTISO, the carotenoid isomerase gene before the branch of the carotenoid biosynthetic pathway, which is expressed in all organs and developmental stages of Chinese kale, and BoaCRTISO, which is located in the chloroplast. The expression of BoaCRTISO is induced by strong light, red and blue combined light, and gibberellic acid treatment, but it is suppressed by darkness and abscisic acid treatment. We obtained BoaCRTISO-silenced plants via virus-induced gene silencing technology, and the silence efficiencies ranged from 52 to $77 \%$. The expressions of most carotenoid and chlorophyll biosynthetic genes in BoaCRTISO-silenced plants were downregulated, and the contents of carotenoids and chlorophyll were reduced. Meanwhile, BoaCRTISOsilenced plants exhibited phenotypes of yellowing leaves and inhibited growth. This functional characterization of BoaCRTISO provides insight for the biosynthesis and regulation of carotenoid in Chinese kale.

Keywords: BoaCRTISO, carotenoid biosynthesis, Chinese kale, color, gene expression, VIGS

\section{INTRODUCTION}

Chinese kale (Brassica oleracea var. alboglabra) is a member of Brassicaceae, or the mustard family, which originated in South China and later spread throughout Southeast Asia (Lei et al., 2017). Its main edible parts are the highly nutritious tender leaves and bolting stems (Sun et al., 2018b). Our previous studies have shown that Chinese kale is rich in health-promoting compounds including vitamin C, glucosinolates, and carotenoids (Sun et al., 2012a,b). Carotenoids are the second largest group of natural pigments and are known to play crucial roles in plant color, photosynthesis, and human health (Xiao et al., 2012). The different types and contents of carotenoids lead to the rich and colorful red, yellow, and orange pigments of vegetables, fruits, and flowers such as tomato (Ye et al., 2015), citrus (Kato et al., 2004), watermelon (Lv et al., 2015), and chrysanthemum (Yamagishi et al., 2010). In green plant tissues, carotenoids are also lightharvesting pigments that are closely associated with chlorophylls in the photosynthetic membranes of plants where they protect chlorophyll against photooxidative damage (Guo et al., 2018; Llorente et al., 2020). In humans, carotenoids are necessary to maintain body health. Carotenoids are the precursors of retinoid synthesis and are theorized to act as antioxidants that reduce the risk of macular degeneration (Nisar et al., 2015). It is also reported that carotenoids could treat chronic 
diseases, such as type 2 diabetes and cardiometabolic diseases, and lower the risk of cancer and cardiovascular disease ( $\mathrm{Gu}$ et al., 2015; Liu et al., 2015). Given the growing list of human health benefits, horticulturists and others have a deep interest in developing carotenoid-rich food crops through breeding and metabolic engineering. Recent research generally uses molecular breeding methods to enhance carotenoids and to focus on changing biosynthetic pathways to alter metabolic flux (Saini et al., 2014; Kaur et al., 2017), such as golden rice (Beyer et al., 2002) and multivitamin corn (Naqvia et al., 2009).

Currently, the carotenoid biosynthetic pathway in complex and vascular plants has been clarified (Nisar et al., 2015). Several major carotenoids in plants appear in their trans form through the activity of isomerases, which are enzymes that function to convert molecules from one isomer to another. For example, carotenoid isomerase (CRTISO) is a key isomerase in the carotenoid biosynthetic pathway, which can catalyze prolycopene to lycopene (Pinheiro et al., 2019). In Arabidopsis (Park et al., 2002), tomato (Isaacson et al., 2002), and cabbage (Su et al., 2015), the loss of CRTISO function could cause the accumulation of pro-lycopene, which delays the greening of Arabidopsis, and changes the tomato color from red to orange and the cabbage color from yellow to orange. A crtiso mutant in rice produces a so-called zebra phenotype with alternating green and chlorotic crossbands on the mutant leaves (Chai et al., 2011). Besides the aesthetic effect on plant color, CRTISO also has a significant functional effect on photosynthetic efficiency. For instance, chlorophyll accumulation during photomorphogenesis was delayed markedly in a ccr2 (Atcrtiso) mutant (Park et al., 2002), while the photosynthetic rate of yellow leaf sectors produced from a crtiso mutant in rice was approximately $27-42 \%$ of that of the wild-type unaffected leaves (Chai et al., 2011). In addition, carotenoid contents and related gene expressions can be regulated by exogenous phytohormone and light treatments in most plants (Divya et al., 2018). The exogenous methyl jasmonate (MeJA) spray treatment significantly enhanced the accumulation of lycopene in tomato (Liu et al., 2012) and other carotenoids in leafy vegetables (Divya et al., 2014; Saini et al., 2014), and abscisic acid (ABA) also had the similar effect in tomato (Sun et al., 2012), whereas gibberellic acid $\left(\mathrm{GA}_{3}\right)$ treatment had a marked effect on preventing the carotenoid accumulation in Navelate oranges (Rodrigo and Zacarias, 2007). It has also been found that the expression levels of BoaPDS1 and BoaPDS2 genes in Chinese kale were both regulated by red and blue combined light, and salicylic acid treatments (Sun et al., 2019). We suggest that these abiotic environmental conditions could regulate carotenoid biosynthetic pathways through CRTISO.

At present, the development of Chinese kale in China is relatively slow compared with other brassica vegetables. One of the key limiting reasons is that its color is not rich enough, and most of the germplasm resources are green (Lei et al., 2017), which makes it very difficult to breed new varieties with other colors via crossbreeding. In this study, we investigated the expression patterns of BoaCRTISO under different conditions; and we obtained BoaCRTISO-silenced plants using virus-induced gene silencing (VIGS) technology, and then we analyzed changes in the phenotype, carotenoid contents, and the related biosynthetic gene expressions of BoaCRTISOsilenced plants. Our findings allow a better understanding of the function of BoaCRTISO in regulating the carotenoid contents and coloring in Chinese kale, and this work will help in the creation of new varieties of Chinese kale from the perspective of molecular breeding.

\section{MATERIALS AND METHODS}

\section{Plant Materials}

In this study, we used the white-flower Chinese kale, Brassica oleracea var. alboglabra "Sijicutiao." The plants were grown in trays containing a mixture of peat and vermiculite (3:1) in an incubator with a light intensity of $80 \mu \mathrm{mol} \mathrm{m} \mathrm{m}^{-2} \mathrm{~s}^{-1}$, a temperature of $25 / 20^{\circ} \mathrm{C}$ (day/night), a $12 / 12$-h (day/night) light cycle, and $75 \%$ humidity. Seedlings with five to six true leaves were transplanted to the greenhouse of Sichuan Agricultural University for cultivation. Fertilizer and water were applied as needed.

Chinese kale materials were sampled at different developmental stages (germinating seeds, cotyledons, fifth to sixth true leaves, and mature leaves), as were organs at different stages of maturation (roots, bolting stems, petioles, leaves, inflorescence, fruit pod, and young fruit), and floral organs at the flower buds stage and the opening flowers stage (sepals, petals, stamens, and pistils). The materials were frozen by liquid nitrogen and stored at $-80^{\circ} \mathrm{C}$ for subsequent studies (Sun et al., 2019).

\section{Light and Phytohormone Treatments}

The 30-day-old Chinese kale seedlings, raised in identical growth conditions, were selected for light intensity, light quality, and phytohormone treatments (Sun et al., 2019). The light quality treatments were divided into red light $(\mathrm{R}: \mathrm{B}=10: 0)$, blue light $(\mathrm{R}: \mathrm{B}=0: 10)$, and red and blue combined light $(\mathrm{R}: \mathrm{B}=5: 5)$ treatments, while white light treatment was used as a control. Light intensity treatments were divided into darkness $(0 \mu \mathrm{mol}$ $\mathrm{m}^{-2} \mathrm{~s}^{-1}$ ), weak light $\left(40 \mu \mathrm{mol} \mathrm{m} \mathrm{m}^{-2} \mathrm{~s}^{-1}\right.$ ), and strong light $\left(120 \mu \mathrm{mol} \mathrm{m} \mathrm{m}^{-2} \mathrm{~s}^{-1}\right)$ groups; the control group was exposed to white light $\left(80 \mu \mathrm{mol} \mathrm{m} \mathrm{m}^{-2} \mathrm{~s}^{-1}\right)$. Phytohormones were sprayed on the leaf surface with $5 \mu \mathrm{M}$ of $\mathrm{GA}_{3}$ and $0.5 \mu \mathrm{M}$ of $\mathrm{ABA}$, and distilled water was used as control. When the leaves ceased dripping, the plants were moved into an artificial intelligence light incubator. In addition, $100 \mu \mathrm{M}$ of MeJA was used to fumigate the Chinese kale seedlings in a transparent and closed container. The cultural conditions of the control and treated Chinese kale seedlings were as described in section "Plant Materials." The fifth to sixth true leaves were sampled at 0,1 , $3,6,12,24,48$, and $72 \mathrm{~h}$ after each treatment, and the samples were immediately frozen in liquid nitrogen and stored at $-80^{\circ} \mathrm{C}$ until RNA extraction.

\section{RNA Extraction and qPCR Analysis}

Total RNA was extracted from Chinese kale using a cetyl trimethylammonium bromide (CTAB) method (Chen et al., 2011). The well-integrated total RNA was used for cDNA 
synthesis using the PrimeScript ${ }^{\mathrm{TM}}$ 1st Strand cDNA Synthesis Kit (TAKARA, Japan). The qPCR primers for carotenoid and chlorophyll biosynthetic genes in Chinese kale were synthesized based on the qPCR primer database ${ }^{1}$, and $\beta$-actin (Büchert et al., 2011) was used as the reference gene (Supplementary Table 1). The qPCR was performed referring to the TB Green Premix Ex Taq II (Tli RNaseH Plus) (TAKARA, Japan) kit instructions by using the Bio-Rad iCycler thermocycler (Bio-Rad, United States). Gene expression analysis was performed using the $2^{-\Delta \Delta C T}$ method (Livak and Schmittgen, 2001).

\section{Molecular Cloning and Sequence Analysis}

According to the sequences of the CRTISO gene and promoters of homologous species such as cabbage and Chinese cabbage published by Brassica database (BRAD), specific primers for the BoaCRTISO gene and promoters were designed (Supplementary Table 1). The method of gene cloning refers to Sun et al. (2018b). The CRTISO amino acid sequence of other species was downloaded from the National Center for Biotechnology Information (NCBI) and subjected to multiple sequence alignment using DNAMAN software (Lynnon Biosoft, United States). The phylogenetic tree was generated using the neighbor-joining method by MEGA 6.0 software (Tamura et al., 2013). The cis-acting elements on the promoter sequences of BoaCRTISO were predicted using the PlantCARE online software ${ }^{2}$.

\section{Subcellular Localization}

Subcellular localization of BoaCRTISO was performed using the methods described by Sun et al. (2018a). The complete coding sequence (CDS) of BoaCRTISO was amplified using the primers BoaCRTISO-GFP-F/R (Supplementary Table 1), in which a BamHI site at the $5^{\prime}$-end and a SpeI site at the $3^{\prime}$-end of the gene were incorporated. The BoaCRTISO and the pC2300-35SeGFP plasmid digested with BamHI and SpeI were mixed to generate pC2300-35S-BoaCRTISO-eGFP plasmid. Chinese kale mesophyll protoplasts were isolated and purified, and then the pC2300-35S-BoaCRTISO-eGFP and pC2300-35S-eGFP plasmids were transformed into Chinese kale protoplasts. The protoplasts expressing green fluorescent protein (GFP)-fusion protein were observed, and images were captured using a BX-51 fluorescence microscope equipped with a DP70 camera (Olympus, Japan).

\section{Virus-Induced Gene Silencing of BoaCRTISO}

A turnip yellow mosaic virus (TYMV)-induced gene silencing system was used to functionally characterize BoaCRTISO according to the previously described methods (Muntha et al., 2018). The pTY vector was digested with SnaBI, and the resulting linearized vector was analyzed by gel electrophoresis to confirm specificity. The linearized pTY vector and the annealed 80base-pair-specific nucleotide of BoaCRTISO were ligated using

${ }^{1}$ https://biodb.swu.edu.cn/qprimerdb/

${ }^{2}$ http://bioinformatics.psb.ugent.be/webtools/plantcare/html/
T4 ligase to form a pTY-BoaCRTISO vector. The amplification of a TYMV-CP gene of the expected size (520 nt) was used to identify positive clones. Details of the pTY-CP F/R primer pairs are provided in Supplementary Table 1. For the virus infiltration, $5 \mu \mathrm{g}$ of purified pTY-S carrying the target gene was diluted in $25 \mu$ l of double-distilled $\mathrm{H}_{2} \mathrm{O}$, which was then used to infiltrate two to four leaves of each plant. The infiltration was repeated every 5 days for 20 days, for a total of four interventions. The control plants were infiltrated with water, and the plants infiltrated with an empty pTY-S vector were used as the reference. Infiltrated plants were incubated in a growth chamber set at $22 / 20^{\circ} \mathrm{C}$ (day/night) with an $8 / 16$-h (light/dark) cycle. One week after the last infiltration, plant phenotype analysis was performed, and the newly grown leaves were sampled for carotenoid composition, content, and related gene expression analysis.

\section{Color}

Color analysis of the transgenic plants was conducted using an NR110 chroma meter ( $3 \mathrm{nh}$, China). Three positions on the sampled leaves of each infiltrated plant were randomly selected, and the color values $L^{*}, a^{*}$, and $b^{*}$ were obtained.

\section{Determination of Carotenoid and Chlorophyll Composition and Contents}

Carotenoid and chlorophyll concentrations were determined using the methods of Sun et al. (2021) with a slight modification. Approximately $200 \mathrm{mg}$ of leaves was ground and extracted with $25 \mathrm{ml}$ of acetone. The samples were sonicated for $20 \mathrm{~min}$ and then centrifuged at $4,000 \mathrm{~g}$ at room temperature for $5 \mathrm{~min}$. The supernatant was filtered through $0.22-\mu \mathrm{m}$ cellulose acetate filters and analyzed by high-performance liquid chromatography (HPLC). HPLC analysis of carotenoids and chlorophyll was carried out using an Agilent 1260 instrument with a VWD detector (Agilent, United States). Samples $(10 \mu \mathrm{l})$ were separated at $30^{\circ} \mathrm{C}$ on a Waters $\mathrm{C} 18$ column $(150 \times 3.9-\mathrm{mm}$ id; 4 $\mu \mathrm{m}$ particle size) using isopropanol and $80 \%$ acetonitrile-water at a flow rate of $0.5 \mathrm{ml} \mathrm{min}-1$. Absorbance was detected at 448 and $428 \mathrm{~nm}$.

\section{Statistical Analysis}

All the results are shown as the means of three replicates. Statistical analysis was performed using SPSS version 18 (SPSS Inc., United States). The data were analyzed using one-way analysis of variance (ANOVA), and differences were compared using the least significant difference (LSD) test at a significance level of 0.05 .

\section{RESULTS}

\section{Isolation and Characterization of the BoaCRTISO}

Analysis of the Brassica oleracea genome data revealed that the CRTISO gene has only one member. The results of cloning of BoaCRTISO also showed that there is only one BoaCRTISO in 

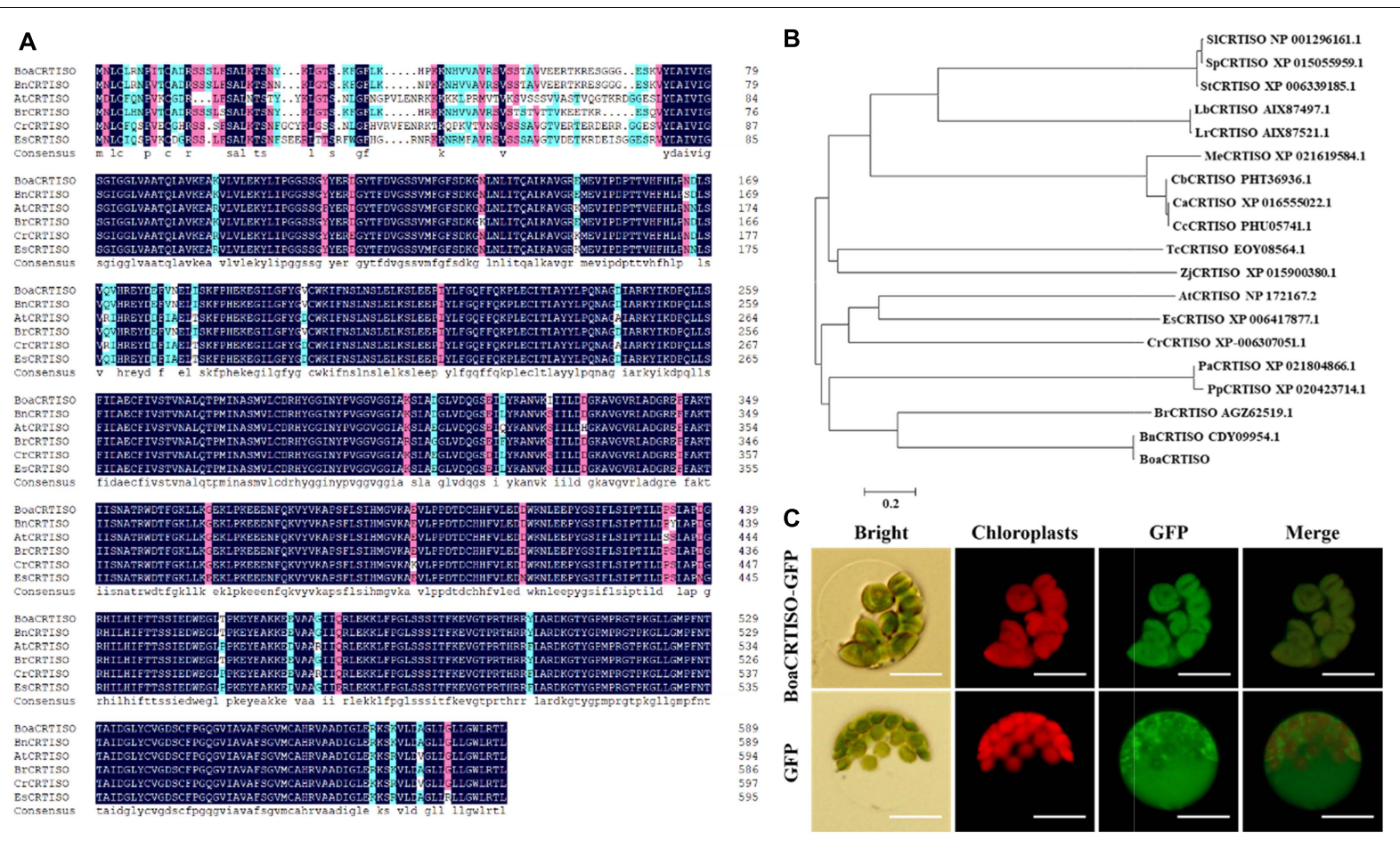

FIGURE 1 | Sequence characteristics of BoaCRTISO. (A) Alignment of the protein sequence of BoaCRTISO with selected homologs. The alignment was performed using DNAMAN software. The amino acids with $100 \%$ identity are shown with a black background, those with $\geq 75 \%$ identity are shown in red, and those with $\geq 50 \%$ identity are shown in blue. The species and their accession numbers in GenBank [Brassica napus (Bn): BnCRTISO (CDY09954.1), Brassica rapa (Br): BrCRTISO (AGZ62519.1), Arabidopsis thaliana (At): AtCRTISO (NP_172167.2), Capsella rubella (Cr): CrCRTISO (XP_006307051.1), and Eutrema salsugineum (Es): ESCRTISO (XP_006417877.1)] are listed here. (B) Phylogenetic analysis of BoaCRTISO and selected CRTISO from other plant species. The phylogenetic tree was generated using the neighbor-joining method by MEGA 6.0 software. The bar indicates an evolutionary distance of $0.2 \%$. The species [B. napus (Bn), B. rapa (Br), A. thaliana (At), C. rubella (Cr), E. salsugineum (Es), Prunus persica (Pp), Lycium barbarum (Lb), Lycium ruthenicum (Lr), Prunus avium (Pa), Manihot esculenta (Me), Ziziphus jujube (Z)), Solanum lycopersicum (SI), Theobroma cacao (Tc), Solanum pennellii (Sp), Solanum tuberosum (St), Capsicum baccatum (Cb), Capsicum annuum (Ca), and Capsicum chinense (Cc)] are listed here. (C) Subcellular localization of the BoaCRTISO-GFP fusion protein in Chinese kale protoplasts. Free green fluorescent protein served as a control. Bars $=30 \mu \mathrm{m}$.

Chinese kale. The CDS of the BoaCRTISO was cloned with sequence lengths of 1,773 bp (GenBank accession MN810158), which encodes a 590-amino-acid protein. Alignment results showed that the changes in amino acid sequences of CRTISO among different species mainly occurred at the N-terminus (Figure 1A). A phylogenetic analysis showed that BoaCRTISO clustered with other Cruciferae CRTISO (Figure 1B), which indicates that CRTISO is highly conserved in cruciferous plants. Moreover, a construct encoding BoaCRTISO fused to GFP was transformed into Chinese kale protoplasts, and strong fluorescence from GFP-BoaCRTISO was detected in the chloroplast (Figure 1C), which clearly demonstrates that BoaCRTISO localizes to the chloroplast.

\section{Temporal and Spatial Expressions of BoaCRTISO}

The BoaCRTISO gene was expressed in all developmental stages and organs (Figure 2). During the development of Chinese kale, the highest level of BoaCRTISO expression was in the cotyledon stage, followed by the germination and maturity stages, and it was lowest in the true leaves stage (Figure 2A). In different organs during the mature period, the expression levels of BoaCRTISO in inflorescences, seed pods, and young seeds were relatively high and were notably more than twice those of other organs (Figure 2B). During the flower buds and opening flowers stages, the expression levels of BoaCRTISO in both pistils and sepals were more than twice those observed in petals and stamens. Compared with the flower buds stage, the expression of BoaCRTISO in all flower organs was significantly downregulated at the opening flowers stage, except for stamens.

\section{Light and Phytohormone Treatments Affected BoaCRTISO Expression}

The promoter of the BoaCRTISO from Chinese kale leaves was cloned with sequence lengths of 1,789 bp (GenBank accession MN810159). The cis-elements of BoaCRTISO promoter mainly contained light- and phytohormone-responsive elements (Figure 3A). The light-responsive elements included AE-box, Box 4, TCT-motif, chs-Unit $1 \mathrm{~m} \mathrm{1,} \mathrm{G-box,} \mathrm{GT1-motif,} \mathrm{and}$ MRE, while phytohormone-responsive elements included ABAresponsive element, MeJA response elements (CGTCA-motif and TGACG-motif), and GA response element (GARE-motif).

Under the treatment of different light intensities, it can be seen that the expression of BoaCRTISO is significantly induced 

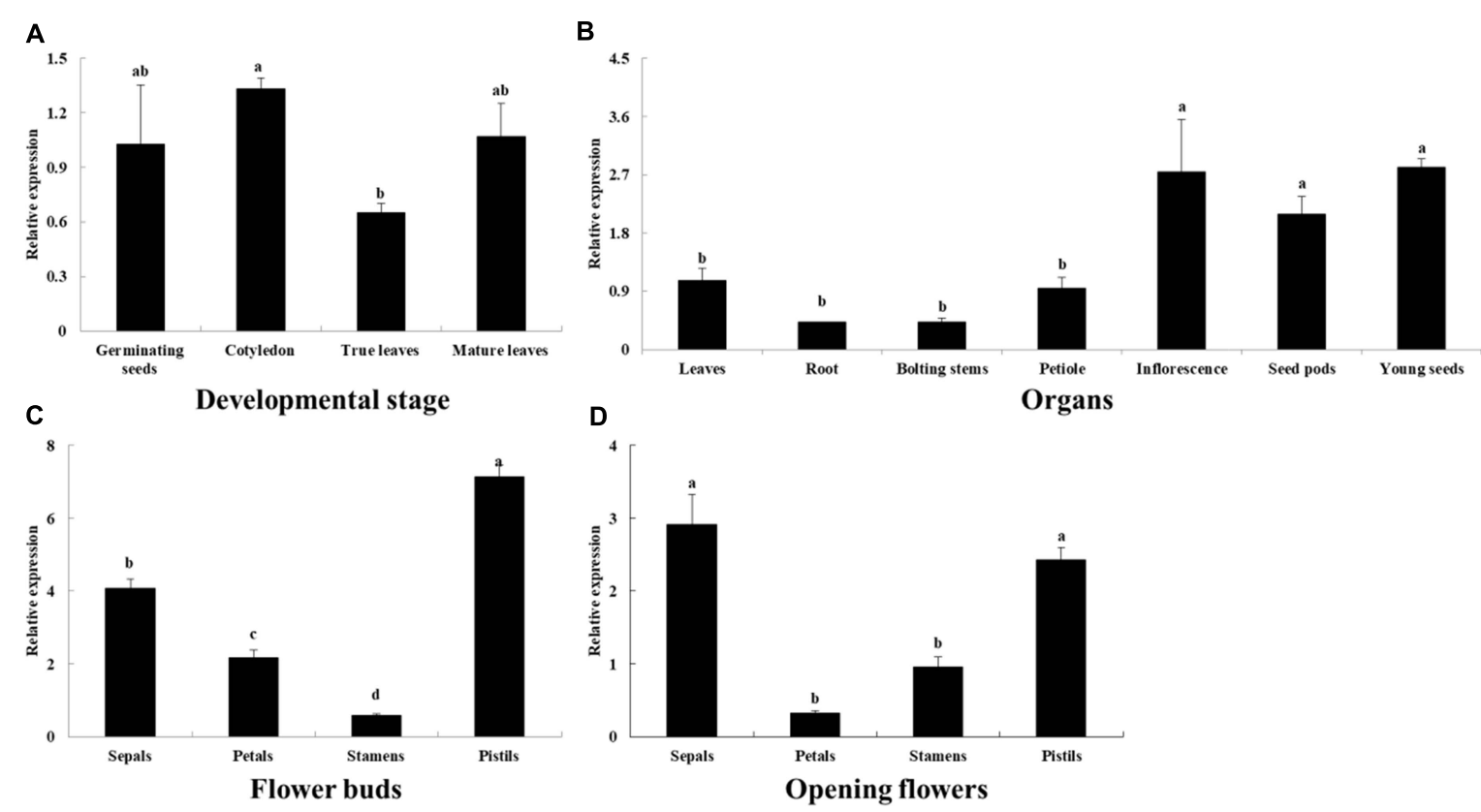

FIGURE 2 | Expression levels of BoaCRTISO in different developmental stages: (A) organs, (B) flower organs in flower buds stage (C), and opening flowers stage (D) of Chinese kale. The BoaCRTISO expression of germinating seeds was set as 1. The samples were a mixture from three individual plants. Data are expressed as mean \pm standard deviation. The same letter in the same histogram indicates that there is no significant difference between the values tested by least significant difference (LSD) $(p<0.05)$.

by strong light and significantly inhibited in the dark, while weak light has little effect on it (Figure 3B and Supplementary Table 2). Under the combined red and blue light treatments, the expression level of BoaCRTISO was significantly higher than that of the control group after $3 \mathrm{~h}$, and its peak value was about three times that of the control group at $6 \mathrm{~h}$. Compared with that of the control group, the expression of BoaCRTISO was significantly lower under red light treatment, but there was no significant difference under blue light treatment (Figure 3C and Supplementary Table 2). Under ABA treatment, the expression level of BoaCRTISO was inhibited before $24 \mathrm{~h}$, but the inhibition gradually weakened afterward (Figure $3 \mathrm{D}$ and Supplementary Table 2). Under $\mathrm{GA}_{3}$ treatment, the expression level of BoaCRTISO was significantly induced (Figure 3E and Supplementary Table 2), while the expression level of BoaCRTISO had no obvious regularity under MeJA treatment (Figure 3F and Supplementary Table 2).

\section{BoaCRTISO Silencing Affected the Color and Inhibited the Growth of Chinese Kale}

The expressions of BoaCRTISO in all pTY-BoaCRTISO plants were significantly reduced compared with control and the pTY plants, except for pTY-BoaCRTISO 1 plant (Figure 4A). Then, we observed that the pTY-BoaCRTISO plants were yellow-green compared with the dark-green of the control and the pTY plants, except for the pTY-BoaCRTISO 1 plant (Figures $\mathbf{4 B}, \mathbf{C}$ ). The changes of color between the pTY-BoaCRTISO plants, control, and pTY plants were then analyzed (Figure 4D). The yellow-blue value $b^{*}$ of pTY-BoaCRTISO 2-6 plants were significantly higher than that of the control and pTY plants, which indicated that pTY-BoaCRTISO 2-6 plants were yellowing. The $b^{*}$ value of the pTY-BoaCRTISO 2 plant with the most remarkable color change was 18 , which was more than three times of that of the control and pTY plants. The red-green value $a^{*}$ of pTY-BoaCRTISO 2-6 plants was significantly lower than that of control and pTY plants. The $L^{*}$ value is similar among different groups. However, the $L^{*}$, $a^{*}$, and $b^{*}$ values of pTY-BoaCRTISO 1 plant were all similar to those of control and pTY plants. In addition, the growth of pTYBoaCRTISO plants were significantly inhibited, and the plant heights were significantly reduced compared with control and pTY plants, except for pTY-BoaCRTISO 1 plant (Figures 4B,E). Therefore, control 1, pTY 3, and pTY-BoaCRTISO 2, 5, and 6 with typical phenotypes and relatively high silencing efficiencies were chosen for subsequent analyses.

\section{BoaCRTISO Silencing Reduced Carotenoids and Chlorophyll Accumulation in Chinese Kale}

After infection with the pTY-BoaCRTISO plasmid, the expression levels of CRTISO of pTY-BoaCRTISO 2, 5, and 6 were significantly reduced and were $23 \%, 45 \%$, and $48 \%$ of the control plants, while the silencing efficiencies were $77 \%, 55 \%$, and 52\%, respectively (Supplementary Figure 1). Next, the total and individual carotenoid contents in pTY-BoaCRTISO plants were analyzed, and we found that their contents were decreased compared with control and pTY plants, except that there was no significant change in $\beta$-carotene (Figure 5). Specifically, lutein, violaxanthin, and neoxanthin in pTY-BoaCRTISO 2 plant were 


\begin{tabular}{llll}
\hline A Function & Cis-element & Sequence(5'-3') & Location of BoaCRTISO \\
\hline Light responsiveness & AE box & AGAAACTT & $-1622(+)$ \\
& chs-Unit 1 m1 & ACCTAACCCGG & $-1076(+)$ \\
& TCT-motif & TCTTAC & $-1655(-)$ \\
& GT1-motif & GGTTAA & $-315(+)$ \\
& Box 4 & ATTAAT & $-1290(+),-554(-),-249(-)$ \\
& G-box & CACGTC & $-1729(+)$ \\
& MRE & AACCTAA & $-52(-)$ \\
Abscisic acid responsiveness & ABRE & AACCCGG & $-1071(+)$ \\
& & ACGTG & $-1725(-)$ \\
& & TACGGTC & $-784(+)$ \\
MeJA responsiveness & CGTCA-motif & CGTCA & $-262(-),-20(-)$ \\
& TGACG-motif & TGACG & $-266(+),-24(+)$ \\
Gibberellin responsiveness & GARE-motif & TCTGTTG & $-277(-)$ \\
\hline
\end{tabular}
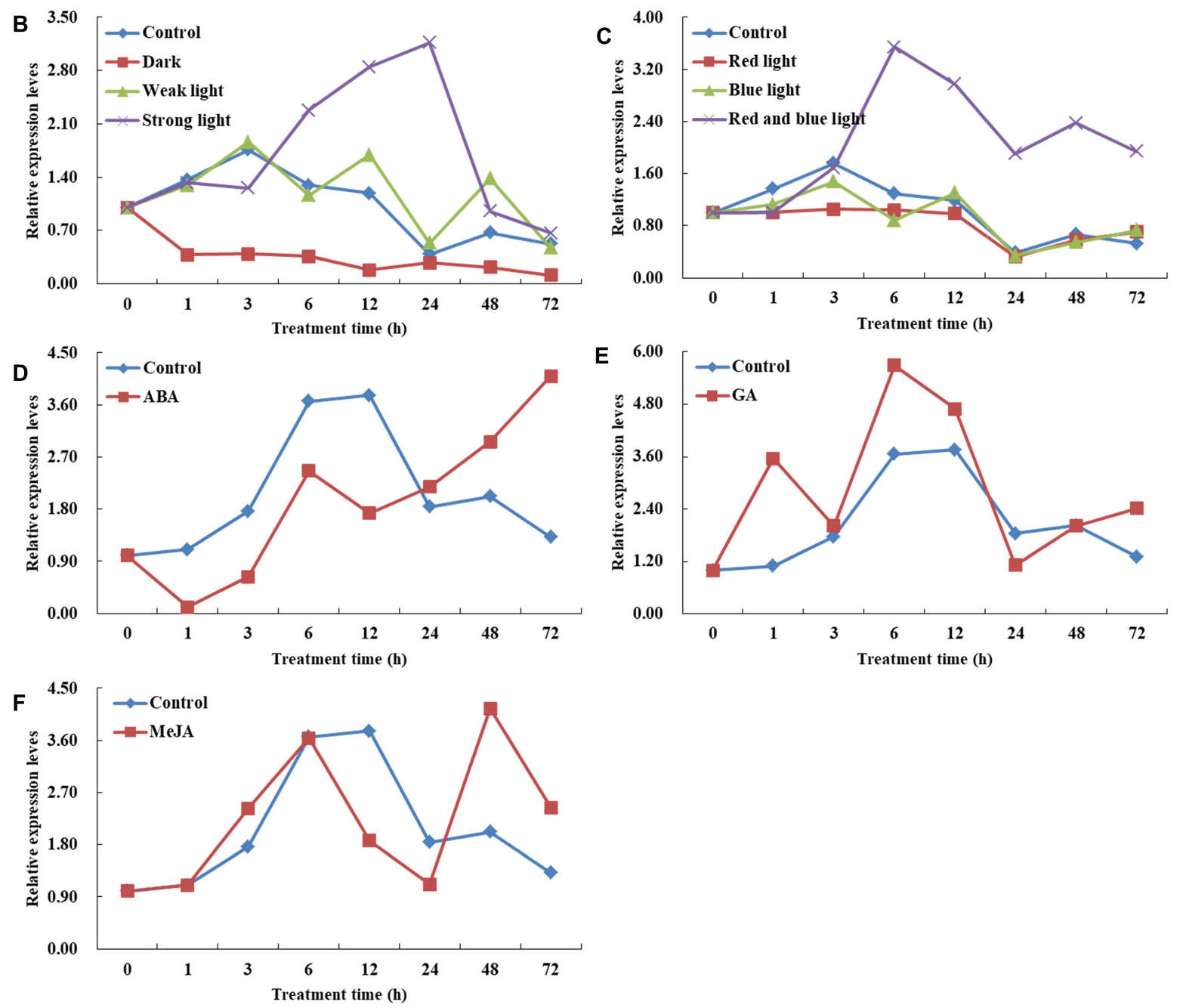

FIGURE 3 | Response of BoaCRTISO to different exogenous treatments. (A) Cis-acting elements respond to light and phytohormone in the promoter regions of BoaCRTISO. The + and - in brackets represent sense strand and antisense strand, respectively. (B) Expression levels of BoaCRTISO after treatments with darkness, weak light, and strong light. (C) Expression levels of BoaCRTISO after treatments with red light, blue light, and combined red and blue lights.

(D) Expression level of BoaCRTISO after abscisic acid (ABA) treatment. (E) Expression level of BoaCRTISO after GA $\mathrm{A}_{3}$ treatment. (F) Expression level of BoaCRTISO after methyl jasmonate (MeJA) treatment. 
A

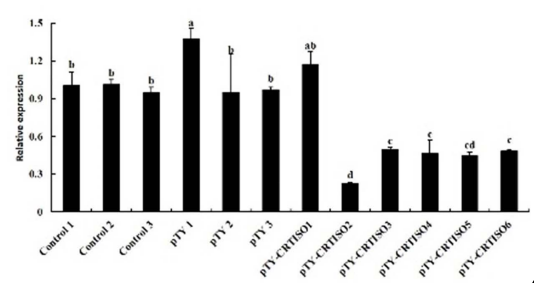

D

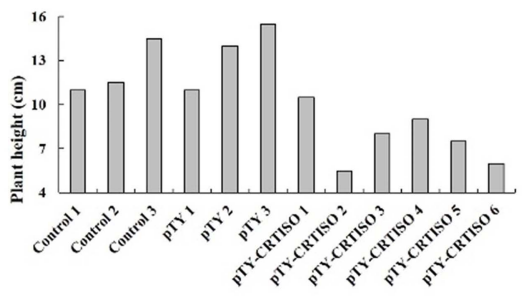

E

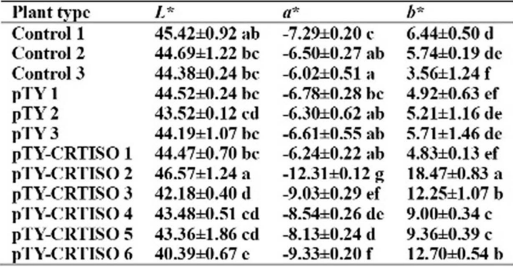

B

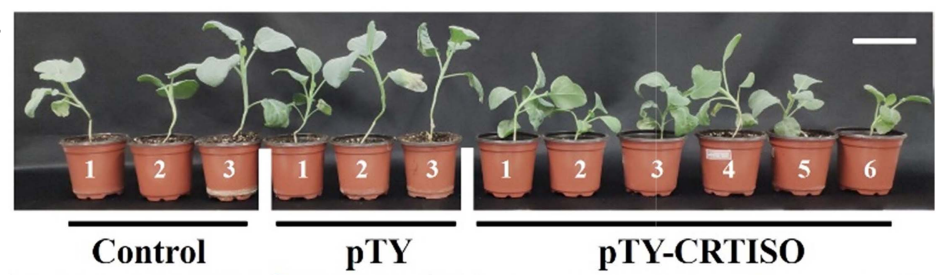

C

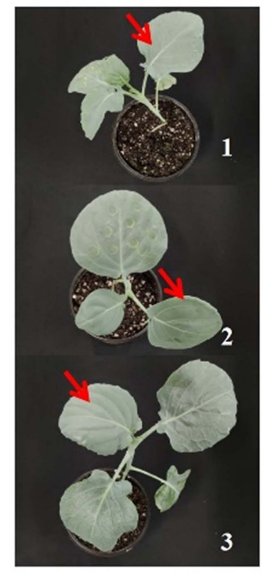

Control

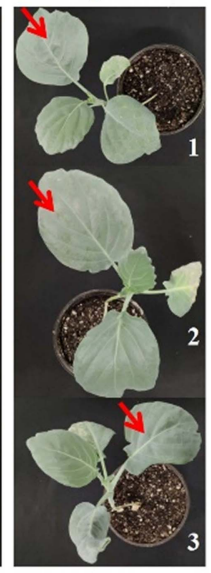

pTY

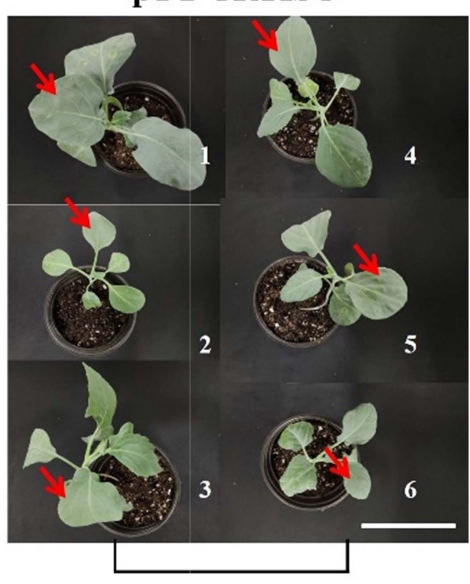

pTY-CRTISO

FIGURE 4 | BoaCRTISO silencing affects the color and growth of Chinese kale. (A) Expressions of BoaCRTISO in control, pTY, and pTY-BoaCRTISO plants. Data are expressed as mean \pm standard deviation. The same letter indicates that there is no significant difference between the values tested by least significant difference (LSD) $(p<0.05)$. (B) Front view of control, pTY, and pTY-BoaCRTISO plants. Bar $=10 \mathrm{~cm}$. (C) Top view of control, pTY, and pTY-BoaCRTISO plants. The red arrow points to the sampled leaves. Bar $=10 \mathrm{~cm}$. (D) Plant heights of control, pTY, and pTY-BoaCRTISO plants at 1 week after the last infiltration. (E) The color parameters of control, pTY, and pTY-BoaCRTISO plants at 1 week after the last infiltration. Data are expressed as a mean \pm SD. The same letter in the same column means no significant differences among values $(p<0.05)$ according to a least significant difference (LSD) test.

only approximately $70 \%$ of the control. Moreover, the contents of lutein, neoxanthin, and violaxanthin in pTY-BoaCRTISO 5 and 6 plants were all reduced, but only significantly so in neoxanthin and lutein. Since lutein content accounted for more than $60 \%$ of the total carotenoid levels in Chinese kale, the total carotenoid contents in pTY-BoaCRTISO 2 and 6 plants were significantly reduced when their lutein contents were significantly inhibited.

Chlorophyll, as a pigment closely related to carotenoids, has also been analyzed for its content. The individual and total chlorophyll of the pTY-BoaCRTISO 2 plant with the lowest BoaCRTISO expression decreased significantly. The changes in chlorophyll of pTY-BoaCRTISO 5 and 6 plants whose expression level of BoaCRTISO decreased by half were different. Chlorophyll $a$ and total chlorophyll in pTY-BoaCRTISO 6 plants decreased significantly, whereas the individual and total chlorophylls of pTY-BoaCRTISO 5 plants did not change significantly.

\section{BoaCRTISO Silencing Suppressed the Expression of Carotenoid and Chlorophyll Biosynthetic Genes}

To investigate whether BoaCRTISO affects carotenoid accumulation by regulating the transcription of carotenoid biosynthetic genes, their expressions in the pTY-BoaCRTISO plants were analyzed by using RT-qPCR (Figure 6 and Supplementary Figure 1). We found that the expression levels of all carotenoid biosynthetic genes in the pTY-BoaCRTISO 2 plant were significantly downregulated except $Z D S$, which was significantly upregulated, and $\beta$-OHase, which was unchanged. Regarding pTY-BoaCRTISO 5 and 6 plants, the expressions of most of their carotenoid biosynthetic genes were also downregulated, while the expressions of ZDS, LCYe2, and $\varepsilon$-OHase genes of pTY-BoaCRTISO 5 plant did not change significantly, and the expressions of ZDS and slightly increased while NXS did not change in the pTY-BoaCRTISO 6 plant.

In pTY-BoaCRTISO plants, chlorophyll, as the main contributor to the color of the leaves of Chinese kale, content decreased significantly, which made us pay attention to the expression of chlorophyll biosynthetic genes. The results indicated that the expression levels of the most chlorophyll biosynthetic genes detected were significantly decreased in the pTY-BoaCRTISO plants (Supplementary Figure 2). These findings suggest that the downregulation of BoaCRTISO expression has an inhibitory effect on the entire carotenoid and chlorophyll biosynthetic pathway in Chinese kale.

\section{DISCUSSION}

The short-duration conventional VIGS has many advantages, including easy and rapid results, unnecessary use of stable plant transformation, and the ability to apply multiple copies or family 

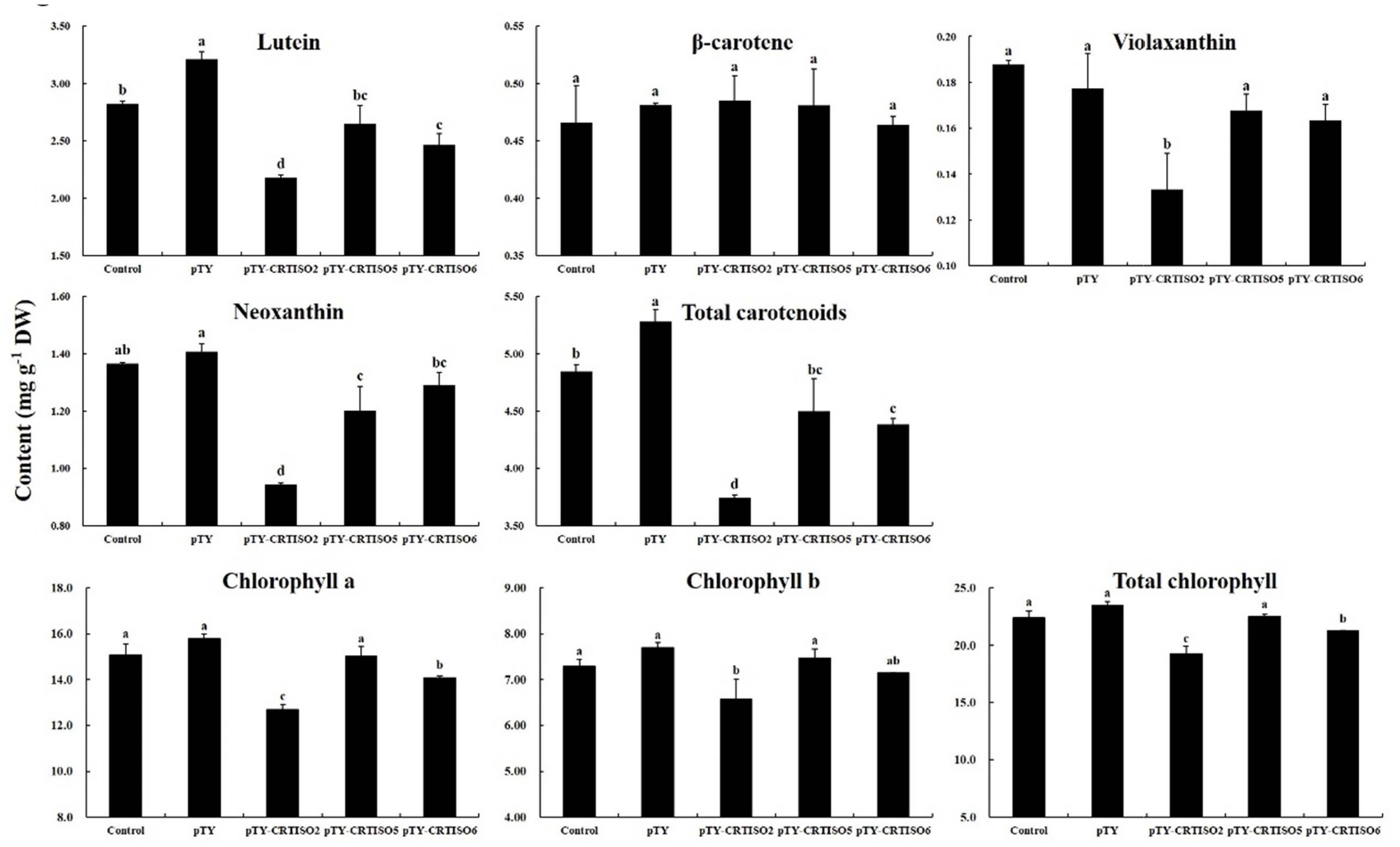

FIGURE 5 | Carotenoid composition and contents in control, pTY, and pTY-BoaCRTISO plants. Samples of leaves were taken from control, pTY, and pTY-BoaCRTISO plants at 1 week after the last infiltration. Data are expressed as mean \pm standard deviation. The same letter in the same histogram indicates that there is no significant difference between the values tested by least significant difference (LSD) $(p<0.05)$.

members (Senthil-Kumar and Mysore, 2011). In this study, the short, inverted repeat coding for BoaCRTISO was inserted into the VIGS system of TYMV to silence the targeted gene, and the resulting BoaCRTISO-silenced plants were successfully obtained with a silencing efficiency of $52-77 \%$. The efficiency of VIGS can be affected by multiple factors, such as crop species and virus types (Senthil-Kumar and Mysore, 2011). Several previous studies indicated that the silencing efficiencies of Brassica juncea, Catharanthus roseus, and strawberry were 90\% (Muntha et al., 2018), 70\% (Kumar et al., 2020), and 45\% (Xie et al., 2020), respectively, which demonstrates the varying degrees of effectiveness across species. We have previously used tobacco rattle virus-mediated VIGS technology for gene silencing in Chinese kale; however, the efficiency of gene silencing was poor (data available upon request). Therefore, we suggest selecting an appropriate virus type when conducting VIGS assays for different crop species.

The role of carotenoids in plant coloration is well studied, and the biosynthetic pathways have been generally described. In this study, it was found that the leaves of Chinese kale color turned yellow and the $b^{*}$ value increased in pTY-BoaCRTISOtreated plants that silenced BoaCRTISO expression. This result is consistent with several previous results but inconsistent with a few others. Our findings are similar to those on seedlings of melons with EMS mutagenesis, which leads to CRTISO being downregulated, or silenced, which appear yellow-green (Galpaz et al., 2013). In a separate study, the leaves showed a yellow-green zebra phenotype in a crtiso mutant in rice (Chai et al., 2011). However, the mutation of CRTISO in tomatoes changes the fruit phenotype to orange (Isaacson et al., 2002). These findings indicate that the phenotypes of leaves and other organs are expressed differently according to species after CRTISO is silenced. Besides, after the BoaCRTISO gene was target-edited in Chinese kale, the plant showed a distinct yellow color, although the gene expression level was similar to that of the VIGS plants (Sun et al., 2020). This shows that stable plant transformation can make plants show a more obvious phenotype than transient VIGS technology. In addition to leaves and fruits, color changes of the floral organs are also related to BoaCRTISO expression. We found that expression of BoaCRTISO in the flower buds stage of Chinese kale was significantly higher than that in the flowering stage, and this finding was contrary to the results of studies on lilies (Yamagishi et al., 2010) and chrysanthemums (Kishimoto and Ohmiya, 2006). This is likely because the Chinese kale flowering process is one of carotenoid degradation, and the opposite process occurs in the chrysanthemum and lily.

After determining that BoaCRTISO partial suppression can yellow Chinese kale leaves, we further explored the relationship among its phenotype, gene expression, and carotenoid contents. Prior work showed that the "Micro Tom" tomato was silenced using an inverted repeat of a fragment from the Citrus CRTISO that resulted in an overall decrease in transcript accumulation of all genes from the carotenoid biosynthetic 


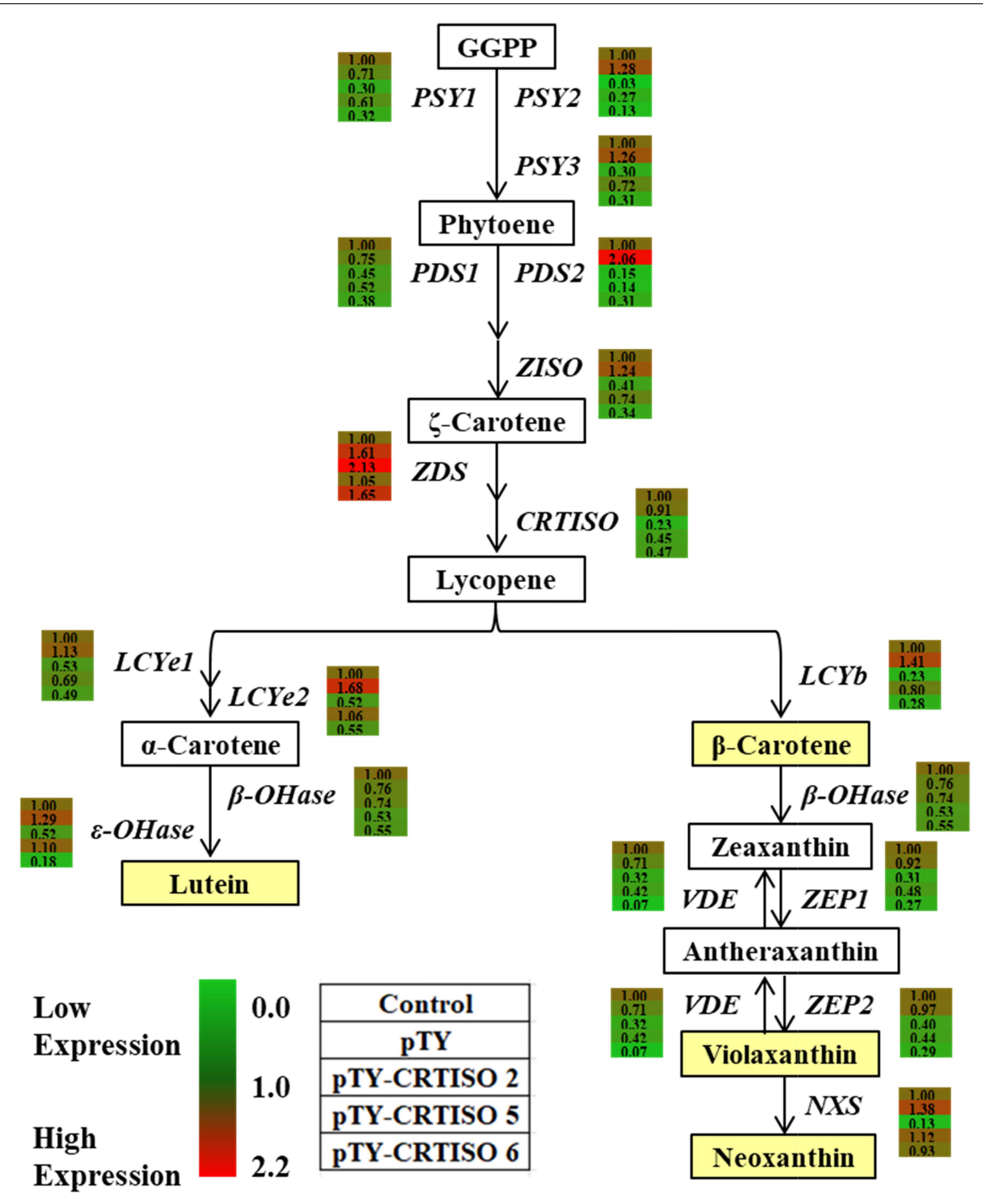

FIGURE 6 | Heat map of carotenoid biosynthetic gene expressions in control, pTY, and pTY-BoaCRTISO plants. Samples of leaves were taken from control, pTY, and pTY-BoaCRTISO plants at 1 week after the last infiltration. GGPP, geranylgeranyl diphosphate; PSY, phytoene synthase; PDS, phytoene desaturase; ZDS, $\zeta$-carotene desaturase; Z-ISO, $\zeta$-carotene isomerase; CRTISO, carotenoid isomerase; LCYe, lycopene $\varepsilon$-cyclase; LCYb, lycopene $\beta$-cyclase; $\varepsilon$-OHase, $\varepsilon$-carotene hydroxylase; $\beta$-OHase, $\beta$-carotene hydroxylase; VDE, violaxanthin de-epoxidase; ZEP, zeaxanthin epoxidase; NXS, neoxanthin synthase.

pathway (Pinheiro et al., 2019), whereas downregulation of CRTISO expression in tomato (Isaacson et al., 2002) and cabbage (Su et al., 2015) can lead to upregulation of upstream gene expression and downregulation of downstream gene expression in the carotenoid biosynthetic pathway, causing the accumulation of pro-lycopene. In our study, we found that after BoaCRTISO expression was downregulated, almost all carotenoid biosynthetic gene expressions in Chinese kale were also downregulated; this finding is consistent with results reported on citrus (Pinheiro et al., 2019) but inconsistent with that known from tomato (Isaacson et al., 2002) and cabbage (Su et al., 2015). Lutein is the primary component of carotenoids in Chinese kale, while prolycopene and lycopene are not detected. Therefore, the downregulation of BoaCRTISO expression led to the overall decline of carotenoid contents rather than the accumulation of prolycopene, as has been reported in other species. These results suggest that CRTISO might have variable functions in different species and that it could regulate the entire suite of carotenoid biosynthetic genes and carotenoid contents in Chinese kale.

We also found that BoaCRTISO expression can be regulated by exogenous light and hormone treatments. In our study, BoaCRTISO expression was induced under strong light, but it was inhibited in darkness compared with the control plants; this indicates that CRTISO is a light-inducible gene, which is consistent with the expression changes of citrus CRTISO under light and dark conditions (Gao et al., 2011). A few previous reports suggested that mixed (red and blue) or white lights could stimulate carotenoid contents in plants in comparison with applications of only red or only blue light (Tamura et al., 2013; Mastropasqua et al., 2020). Similarly, 
we found that the red and blue combination light treatment significantly induced BoaCRTISO expression compared with applications of only red, blue, or white light. In addition to light, BoaCRTISO expression was also regulated by $\mathrm{ABA}, \mathrm{GA}_{3}$, and MeJA exogenous treatments. After ABA treatment, we observed that BoaCRTISO expression decreases significantly in the early stage; this may be due to the effect of feedback inhibition where endogenous ABA decreased because the content of exogenous ABA increased, and then the biosynthesis of carotenoids (which are an ABA precursor) decreased accordingly. In a similar fashion, it has been reported that gibberellin synthesis is inhibited after overexpression of a lycopene $\beta$-cyclase gene in tobacco, which indicates that gibberellin could negatively regulate carotenoid biosynthesis (Moreno et al., 2016). However, we found that BoaCRTISO expression was positively regulated by $\mathrm{GA}_{3}$ treatment, which may be because the tested carotenoid biosynthetic genes differed in the previous study. In addition, MeJA has a dosage effect of high concentration inhibition and low concentration promotion on the regulation of carotenoids in tomato (Liu et al., 2012), which is consistent with our results. These results suggest that external environmental changes could be manipulated to regulate carotenoid biosynthesis.

Meanwhile, CRTISO plays an important role in photosynthesis and plant growth (Xie et al., 2019). In rice, the reduced photosynthetic rate of crtiso mutant leaves proved that CRTISO has an effect on photosynthesis (Chai et al., 2011). In our study, the expression of BoaCRTISO in leaves was significantly higher than that in root organs, and the subcellular localization of BoaCRTISO was in the chloroplast; this is a key organelle for photosynthesis, and the localization we observed may have occurred because leaves are the main photosynthetic organs and require more carotenoids than other plant tissues (Nisar et al., 2015). These results proved that BoaCRTISO plays an important role in photosynthesis by Chinese kale. In addition, the temporal and spatial expression patterns of BoaCRTISO were constitutive, and the heights of pTY-BoaCRTISO 2, 5, and 6 plants were significantly reduced, which indicates that BoaCRTISO is a necessary gene for the growth and development of Chinese kale. When the expression of carotenoid biosynthetic genes decreased, the growth and development of fleshy roots of carrots were also restricted (Flores-Ortiz et al., 2020), which is consistent with our results. In addition, the chlorophyll content and biosynthetic gene expressions in pTY-BoaCRTISO plants were significantly reduced, which would also affect photosynthesis to inhibit plant growth. This suggested that BoaCRTISO may affect plant growth by directly or indirectly affecting photosynthesis; nevertheless, the specific mechanism needs further study.

In summary, the carotenoid isomerase gene BoaCRTISO and promoter of Chinese kale were cloned, and BoaCRTISO was located in the chloroplast via a subcellular localization assay. The expression of BoaCRTISO was detected in all growth and development periods, and organs, of Chinese kale. VIGS-mediated BoaCRTISO silencing suppressed the expression of carotenoid and chlorophyll biosynthetic genes, decreased carotenoid and chlorophyll contents, yellowed Chinese kale, and inhibited Chinese kale growth. In addition, BoaCRTISO expression can be induced by strong light, combined red and blue light, and $\mathrm{GA}_{3}$ treatments but inhibited by darkness and ABA treatments. These findings collectively mean that BoaCRTISO is a viable gene to target for improvements of the appearance and nutritional quality of Chinese kale for the betterment of human health.

\section{DATA AVAILABILITY STATEMENT}

The original contributions presented in the study are included in the article/Supplementary Material, further inquiries can be directed to the corresponding authors.

\section{AUTHOR CONTRIBUTIONS}

MJ: investigation, data curation, and writing - original draft. FZ: methodology and writing - original draft. QY and PL: data curation. HZ, SL, and YJ: investigation. HM: methodology and data curation. QW and HL: conceptualization, funding acquisition, and writing - review and editing. BS: conceptualization, funding acquisition, writing - review and editing, and supervision. All authors review and editing.

\section{FUNDING}

This work was supported by the National Natural Science Foundation of China (32072586 and 31500247), Zhejiang Provincial Ten-thousand Program for Leading Talents of Science and Technology Innovation (2018R52026), Sichuan Science and Technology Program (2018NZ0081), Project of New Varieties Breeding of Sichuan Vegetable Innovation Team (sccxtd-202005), and Technology Project of Zhishi Supply Chain Technology Co., Ltd. (2020008).

\section{SUPPLEMENTARY MATERIAL}

The Supplementary Material for this article can be found online at: https://www.frontiersin.org/articles/10.3389/fpls.2021. 662684/full\#supplementary-material

Supplementary Figure 1 | Expressions of carotenoid biosynthetic genes in control, pTY, and pTY-BoaCRTISO plants. Samples of leaves were taken from control, pTY, and pTY-BoaCRTISO plants at 1 week after the last infiltration. The carotenoid biosynthetic gene expressions were calculated based on the respective expression of the respective genes in control plant. Data are expressed as mean \pm standard deviation. The same letter in the same histogram indicates that there is no significant difference between the values tested by LSD $(p<0.05)$.

Supplementary Figure 2 | Expressions of chlorophyll biosynthetic genes in control, pTY, and pTY-BoaCRTISO plants. Samples of leaves were taken from control, pTY, and pTY-BoaCRTISO plants at 1 week after the last infiltration. The chlorophyll biosynthetic gene expressions were calculated based on the respective expression of the respective genes in control plant. Data are expressed as mean \pm standard deviation. The same letter in the same histogram indicates that there is no significant difference between the values tested by LSD $(p<0.05)$. ALAD, 5-aminolevulinic acid dehydratase; HemE1, glutamyl tRNA reductase; Chl, magnesium-chelatase; CS, chlorophyll synthase. 


\section{REFERENCES}

Beyer, P., Al-Babili, S., Ye, X. D., Lucca, P., Schaub, P., Welsch, R., et al. (2002). Golden rice: introducing the $\beta$-carotene biosynthesis pathway into rice endosperm by genetic engineering to defeat vitamin a deficiency. J. Nutr. 2, 506S-510S.

Büchert, A. M., Civello, P. M., and Martínez, G. A. (2011). Effect of hot air, UV-C, white light and modified atmosphere treatments on expression of chlorophyll degrading genes in postharvest broccoli (Brassica oleracea L.) florets. Sci. Hortic. 127, 214-219. doi: 10.1016/j.scienta.2010.11.001

Chai, C. L., Fang, J., Liu, Y., Tong, H. N., Gong, Y. Q., Wang, Y. Q., et al. (2011). ZEBRA2, encoding a carotenoid isomerase, is involved in photoprotection in rice. Plant Mol. Biol. 75, 211-221. doi: 10.1007/s11103-010-9719-Z

Chen, Q., Yu, H. W., Wang, X. R., Xie, X. L., Yue, X. Y., and Tang, H. R. (2011). An alternative cetyltrimethylammonium bromide-based protocol for RNA isolation from blackberry (Rubus L.). Genet. Mol. Res. 11, 1773-1782. doi: 10.4238/2012.june.29.10

Divya, P., Puthusseri, B., and Neelwarne, B. (2014). The effect of plant regulators on the concentration of carotenoids and phenolic compounds in foliage of coriander. LWT Food Sci. Technol. 56, 101-110. doi: 10.1016/j.lwt.2013.11.012

Divya, P., Puthusseri, B., Lokesh, M. A., and Neelwarne, B. (2018). Effects of methyl jasmonate and carotenogenic inhibitors on gene expression and carotenoid accumulation in coriander (Coriandrum sativum L.) foliage. Food Res. Int. 111, 11-19. doi: 10.1016/j.foodres.2018.04.040

Flores-Ortiz, C., Alvarez, L. M., Undurraga, A., Arias, D., Durán, F., Wegener, G., et al. (2020). Differential role of the two $\zeta$-carotene desaturase paralogs in carrot (Daucus carota): ZDS1 is a functional gene essential for plant development and carotenoid synthesis. Plant Sci. 291:110327. doi: 10.1016/j.plantsci.2019.110327

Galpaz, N., Burger, Y., Lavee, T., Tzuri, G., Sherman, A., Melamed, T., et al. (2013). Genetic and chemical characterization of an EMS induced mutation in Cucumis melo CRTISO gene. Arch. Biochem. Biophys. 539, 117-125. doi: 10.1016/j.abb. 2013.08.006

Gao, H. J., Xu, J., Liu, X., Giuliano, G., Batschauer, A., and Kleinig, H. (2011). Light effect on carotenoids production and expression of carotenogenesis genes in citrus callus of four genotypes. Acta Physiol. Plant. 33, 2485-2492. doi: 10.1007/s11738-011-0793-x

Gu, H. H., Wang, J. S., Zhao, Z. Q., Sheng, X. G., Yu, H. F., and Huang, W. B. (2015). Characterization of the appearance, health promoting compounds, and antioxidant capacity of the florets of the loose-curd cauliflower. Int. J. Food Prop. 18, 392-402. doi: 10.1080/10942912.2013.831445

Guo, X. Y., Song, C. K., Ho, C. T., and Wan, X. C. (2018). Contrbution of L-theanine to the formation of 2, 5-dimetheylpyrazine, a key roasted peanutty flavor in Oolong tea during manufacturing processes. Food Chem. 263, 18-28. doi: 10.1016/j.foodchem.2018.04.117

Isaacson, T., Ronen, G., Zamir, D., and Hirschberg, J. (2002). Cloning of tangerine from tomato reveals a carotenoid isomerase essential for the production of $\beta$ carotene and xanthophylls in plants. Plant Cell 14, 333-342. doi: 10.1105/tpc. 010303

Kato, M., Ikoma, Y., Matsumoto, H., Sugiura, M., Hyodo, H., and Yano, M. (2004). Accumulation of carotenoids and expression of carotenoid biosynthetic genes during maturation in citrus fruit. Plant Physiol. 134, 824-837. doi: 10.1104/pp. 103.031104

Kaur, N., Pandey, A. S., Kumar, P., Pandey, P., Kesarwani, A. K., Mantri, S. S., et al. (2017). Regulation of banana phytoene synthase (MaPSY) expression, characterization and their modulation under various abiotic stress conditions. Front. Plant Sci. 8:462.

Kishimoto, S., and Ohmiya, A. (2006). Regulation of carotenoid biosynthesis in petals and leaves of chrysanthemum (Chrysanthemum morifolium). Physiol. Plant 128, 437-447.

Kumar, S. R., Rai, A., Bomzan, D. P., Kumar, K., Hemmerlin, A., Dwivedi, V., et al. (2020). A plastid-localized bona fide geranylgeranyl diphosphate synthase plays a necessary role in monoterpene indole alkaloid biosynthesis in Catharanthus roseus. Plant J. 103, 248-265. doi: 10.1111/tpj.14725

Lei, J. J., Chen, G. J., Chen, C. M., and Cao, B. H. (2017). Germplasm diversity of Chinese kale in China. Hortic. Plant J. 3, 101-104. doi: 10.1016/j.hpj.2017. 07.006

Liu, L. H., Shao, Z. Y., Zhang, M., and Wang, Q. M. (2015). Regulation of carotenoid metabolism in tomato. Mol. Plant 8, 28-39. doi: 10.1016/j.molp. 2014.11.006
Liu, L. H., Wei, J., Zhang, M., Zhang, L. P., Li, C. Y., and Wang, Q. M. (2012). Ethylene independent induction of lycopene biosynthesis in tomato fruits by jasmonates. J. Exp. Bot. 63, 5751-5761. doi: 10.1093/jxb/ers224

Livak, K. J., and Schmittgen, T. D. (2001). Analysis of relative gene expression data using real-time quantitative PCR and the 2- $\Delta \Delta$ CT method. Methods 25, 402-408. doi: 10.1006/meth.2001.1262

Llorente, B., Torres-Montilla, S., Morelli, L., Florez-Sarasa, I., Matus, J. T., Ezquerro, M., et al. (2020). Synthetic conversion of leaf chloroplasts into carotenoid-rich plastids reveals mechanistic basis of natural chromoplast development. P. Natl. Acad. Sci. USA 117, 21796-21803. doi: 10.1073/pnas. 2004405117

Lv, P., Li, N., Liu, H., Gu, H. H., and Zhao, W. E. (2015). Changes in carotenoid profiles and in the expression pattern of the genes in carotenoid metabolisms during fruit development and ripening in four watermelon cultivars. Food Chem. 174, 52-59. doi: 10.1016/j.foodchem.2014.11.022

Mastropasqua, L., Dipierro, N., and Paciolla, C. (2020). Effects of darkness and light spectra on nutrients and pigments in Radish, Soybean, Mung Bean and Pumpkin Sprouts. Antioxidants 9:558. doi: 10.3390/antiox9060558

Moreno, J. C., Cerda, A., Simpson, K., Lopez-Diaz, I., Carrera, E., Handford, M., et al. (2016). Increased Nicotiana tabacum fitness through positive regulation of carotenoid, gibberellin and chlorophyll pathways promoted by Daucus carota lycopene $\beta$-cyclase (Dclcyb1) expression. J. Exp. Bot. 67, 2325-2338. doi: 10. 1093/jxb/erw037

Muntha, S. T., Zhang, L. L., Zhou, Y. F., Zhao, X., Hu, Z. Y., Yang, J. H., et al. (2018). Phytochrome a signal transduction 1 and CONSTANS-LIKE 13 coordinately orchestrate shoot branching and flowering in leafy Brassica juncea. Plant Biotechnol. J. 17:13057.

Naqvia, S., Zhua, C. F., Farrea, G., Ramessara, K., Bassiea, L., Breitenbachb, J., et al. (2009). Transgenic multivitamin corn through biofortification of endosperm with three vitamins representing three distinct metabolic pathways. P. Natl. Acad. Sci. USA. 106, 7762-7767. doi: 10.1073/pnas.0901412106

Nisar, N., Li, L., Lu, S., Khin, N. C., and Pogson, B. J. (2015). Carotenoid metabolism in plants. Mol. Plant 8, 68-82. doi: 10.1016/j.molp.2014.12.007

Park, H., Kreunen, S. S., Cuttriss, A. J., DellaPenna, D., and Pogson, B. J. (2002). Identification of the carotenoid isomerase provides insight into carotenoid biosynthesis, prolamellar body formation, and photomorphogenesis. Plant Cell 14, 321-332. doi: 10.1105/tpc.010302

Pinheiro, T. T., Peres, L. E. P., Purgatto, E., Latado, R. R., Maniero, R. A., Martins, M. M., et al. (2019). Citrus carotenoid isomerase gene characterization by complementation of the "Micro-Tom" tangerine mutant. Plant Cell Rep. 38, 623-636. doi: 10.1007/s00299-019-02393-2

Rodrigo, M. J., and Zacarias, L. (2007). Effect of postharvest ethylene treatment on carotenoid accumulation and the expression of carotenoid biosynthetic genes in the flavedo of orange (Citrus sinensis L. Osbeck) fruit. Postharvest Biol. Tech. 43, 14-22.

Saini, R. K., Prashanth, K. V. H., Shetty, N. P., and Giridhar, P. (2014). Elicitors, SA and MJ enhance carotenoids and tocopherol biosynthesis and expression of antioxidant related genes in Moringa oleifera Lam. leaves. Acta Physiol. Plant. 36, 2695-2704. doi: 10.1007/s11738-014-1640-7

Senthil-Kumar, M., and Mysore, K. S. (2011). New dimensions for VIGS in plant functional genomics. Trends Plant Sci. 16, 656-665. doi: 10.1016/j.tplants.2011. 08.006

Su, T. B., Yu, S. C., Wang, J., Zhang, F. L., Yu, Y. J., Zhang, D. S., et al. (2015). Loss of function of the carotenoid isomerase gene BrCRTISO confers orange color to the inner leaves of Chinese cabbage (Brassica rapa L. ssp. pekinensis). Plant Mol. Biol. Rep. 33, 648-659. doi: 10.1007/s11105-014-0779-0

Sun, B., Di, H. M., Zhang, J. Q., Xia, P. X., Huang, W. L., Jian, Y., et al. (2021). Effect of light on sensory quality, health-promoting phytochemicals and antioxidant capacity in post-harvest baby mustard. Food Chem. 339:128057. doi: 10.1016/j. foodchem.2020.128057

Sun, B., Jiang, M., Liang, S., Zheng, H., Chen, Q., Wang, Y., et al. (2019). Functional differences of BaPDS1 and BaPDS2 genes in Chinese kale. Roy. Soc. Open Sci. 6:190260. doi: 10.1098/rsos.190260

Sun, B., Jiang, M., Zheng, H., Jian, Y., Huang, W. L., Yuan, Q., et al. (2020). Color-related chlorophyll and carotenoid concentrations of Chinese kale can be altered through CRISPR/Cas9 targeted editing of the carotenoid isomerase gene BoaCRTISO. Hortic. Res. 7:161.

Sun, B., Yan, H. Z., Liu, N., Wei, J., and Wang, Q. M. (2012a). Effect of 1-MCP treatment on postharvest quality characters, antioxidants and glucosinolates 
of Chinese kale. Food Chem. 131, 519-526. doi: 10.1016/j.foodchem.2011. 09.016

Sun, B., Yan, H. Z., Zhang, F., and Wang, Q. M. (2012b). Effects of plant hormones on main health-promoting compounds and antioxidant capacity of Chinese kale. Food Res. Int. 48, 359-366. doi: 10.1016/j.foodres.2012.04.021

Sun, B., Zhang, F., Xiao, N., Jiang, M., Yuan, Q., Xue, S. L., et al. (2018a). An efficient mesophyll protoplast isolation, purification and PEG-mediated transient gene expression for subcellular localization in Chinese kale. Sci. Hortic. 241, 187-193. doi: 10.1016/j.scienta.2018.07.001

Sun, B., Zhang, F., Xue, S. L., Chang, J. Q., Zheng, A. H., Jiang, M., et al. (2018b). Molecular Cloning and Expression Analysis of the $\zeta$-Carotene Desaturase Gene in Chinese kale (Brassica oleracea var. alboglabra Bailey). Hortic. Plant J. 4, 94-102. doi: 10.1016/j.hpj.2018.03.005

Sun, L., Yuan, B., Zhang, M., Wang, L., Cui, M., Wang, Q., et al. (2012). Fruitspecific RNAi-mediated suppression of SINCED1 increases both lycopene and beta-carotene contents in tomato fruit. J. Exp. Bot. 63, 3097-3108. doi: 10.1093/ jxb/ers026

Tamura, K., Stecher, G., Peterson, D., Filipski, A., and Kumar, S. (2013). MEGA6: molecular evolutionary genetics analysis version 6.0. Mol. Biol. Evol. 30, 27252729. doi: $10.1093 / \mathrm{molbev} / \mathrm{mst} 197$

Xiao, Z. L., Lester, G. E., Luo, Y. G., and Wang, Q. (2012). Assessment of vitamin and carotenoid concentrations of emerging food products: edible microgreens. J. Agric. Food Chem. 60, 7644-7651. doi: 10.1021/jf300459b

Xie, J., Yao, S. X., Ming, J., Deng, L. L., and Zeng, K. F. (2019). Variations in chlorophyll and carotenoid contents and expression of genes involved in pigment metabolism response to oleocellosis in citrus fruits. Food Chem. 272, 49-57. doi: 10.1016/j.foodchem.2018.08.020

Xie, Y. G., Ma, Y. Y., Bi, P. P., Wei, W., Li, J., Hu, Y., et al. (2020). Transcription factor FvTCP9 promotes strawberry fruit ripening by regulating the biosynthesis of abscisic acid and anthocyanins. Plant Physiol. Biochem. 146, 374-383. doi: 10.1016/j.plaphy.2019.11.004

Yamagishi, M., Kishimoto, S., and Nakayama, M. (2010). Carotenoid composition and changes in expression of carotenoid biosynthetic genes in tepals of Asiatic hybrid lily. Plant Breed. 129, 100-107. doi: 10.1111/j.1439-0523.2009.01656.x

Ye, J. H., T. X., Yang, C. M., Li, H. X., Yang, M. Z., et al. (2015). Transcriptome profiling of tomato fruit development reveals transcription factors associated with ascorbic acid, carotenoid and flavonoid biosynthesis. PLoS One 10:e0130885. doi: 10.1371/journal.pone.0130885

Conflict of Interest: The authors declare that the research was conducted in the absence of any commercial or financial relationships that could be construed as a potential conflict of interest.

Copyright (c) 2021 Jiang, Zhang, Yuan, Lin, Zheng, Liang, Jian, Miao, Li, Wang and Sun. This is an open-access article distributed under the terms of the Creative Commons Attribution License (CC BY). The use, distribution or reproduction in other forums is permitted, provided the original author(s) and the copyright owner(s) are credited and that the original publication in this journal is cited, in accordance with accepted academic practice. No use, distribution or reproduction is permitted which does not comply with these terms. 